\title{
Evaluation of malnutrition in hospitalised patients in Greece
}

\author{
G. Marakis, T. Karagiozoglou, I. Votsi, E. Kosteletou, L. Kontopoulou and F. Tsofliou \\ Laboratory of Clinical Nutrition, Department of Human Nutrition and Dietetics, Technological Educational Institute \\ of Thessaloniki, Thessaloniki, Greece
}

\begin{abstract}
Malnutrition is prevalent among hospitalised patients worldwide ${ }^{(1)}$. Nutrition screening is not routinely performed among patients in hospitals in Greece, partly because of a confusion that exists about the reliability and practicality of various screening tools.

The present preliminary study aimed at evaluating, for the first time in Greece, the use of two nutrition screening tools for the assessment of the risk of undernutrition in hospitalised patients. Undernutrition risk score (URS) ${ }^{(2)}$ and nutritional risk screening (NRS$2002)^{(3)}$ were simultaneously used for the nutrition screening of seventy-one patients in a pathology clinic (age 49 (SD 17) years, BMI 25.7 $(\mathrm{SD} 4.8) \mathrm{kg} / \mathrm{m}^{2}$ ) and thirty-one patients in an orthopaedic clinic (age 50 (SD 20) years, BMI 26 (SD 5.2) kg/m²) of Papageorgiou Hospital (Thessaloniki, Greece).

The URS screening tool classified $11.3 \%$ of patients in the pathology clinic and $9.7 \%$ of the patients in the orthopaedic clinic as at severe risk of undernutrition. NRS-2002 identified only $1 \%$ of patients in the pathology clinic as at severe risk of undernutrition. There was a significant difference between the two tools (ANOVA) in the pathology clinic $(P<0.001)$ as well as in the orthopaedic clinic $(P=0.009)$. Significant positive correlations were found between the total score of URS and percentage body weight loss $(r 0.7, P<0.001)$ and between total score of NRS-2002 and percentage body weight loss $(r 0.6 ; P<0.001)$, while significant negative correlations were found between total score URS and triceps skinfold thickness $(r-0.37, P=0.002)$ and BMI $(r-0.31, P=0.008)$ for patients in the pathology clinic.

URS appears to be more effective in identifying patients who are at severe risk of malnutrition compared with NRS-2002. The URS screening tool is significantly associated with nutritional indices.
\end{abstract}

1. McWhirter JP \& Pennington CR (1994) Br Med J 9, 945-948.

2. Doyle MP, Barnes E \& Moloney M (2000) J Hum Nutr Diet 13, 433-444.

3. Kondrup J, Rasmussen HH, Hamberg O et al. (2003) Clin Nutr 22, 321-336. 